\title{
On a conjecture of Yiming Long
}

by

\author{
Chaohua Jia (Beijing)
}

1. Introduction. In 2000 when studying the Maslov-type index theory for Hamiltonian systems, Yiming Long [1] put forward the following two conjectures.

Conjecture 1. For any positive integer $k$, there are infinitely many pairs of prime numbers which are of the form $(k n-1, k n+1)$ with a positive integer $n$.

CONJECTURE 2. For any irrational number $\varphi$ in the interval $(0,1)$, there are infinitely many prime numbers $p$ which cannot be expressed as

$$
p=2 n+2[n \varphi]+1
$$

with a positive integer $n$.

In January 2004, Professor Yiming Long proposed his conjectures to me. From the viewpoint of Diophantine equations Conjecture 1 seems as difficult as the prime twins conjecture. In this paper, we shall give a positive answer to Conjecture 2. In fact, we can get more information.

In the following, we suppose that $\varphi$ is an irrational number in the interval $(0,1)$ and that

$$
\alpha=-\frac{1}{2(1+\varphi)},
$$

which is also irrational. By a well known result of Dirichlet (see page 9 of [4]), there are infinitely many rational numbers $a / q((a, q)=1, q \rightarrow \infty)$ such that $|\alpha-a / q| \leq 1 / q^{2}$. We suppose that $q$ is sufficiently large and that $\varepsilon$ is a sufficiently small positive constant, $\delta=\varepsilon^{2}$. Write $((x))=x-[x]-1 / 2$ and $e(x)=e^{2 \pi i x}$. Let $\|y\|$ denote the smallest distance from $y$ to integers, $p$ a prime number and $\Lambda(n)$ the Mangoldt function.

On the above supposition, we have

2000 Mathematics Subject Classification: 11P32, 11L20. 
TheOREM. Let $T(p)$ be the number of solutions of the equation (1) in positive integers $n$. If $q^{1+\varepsilon}<x \leq q^{1 / \varepsilon}$, then

$$
\sum_{\substack{x<p \leq 2 x \\ T(p)=0}} 1 \geq \frac{\varphi-\varepsilon}{1+\varphi} \cdot \frac{x}{\log x} .
$$

We see that not only Conjecture 2 is true but also in a lot of intervals the relevant prime numbers have a positive density by the fact that

$$
\sum_{x<p \leq 2 x} 1 \sim \frac{x}{\log x} .
$$

2. Proof of the Theorem. For a prime number $p \geq 3$, the following equivalences hold:

$$
\begin{aligned}
2 n+2[n \varphi] & +1=p, n>0 \Leftrightarrow[n \varphi]=\frac{p-1}{2}-n, n>0 \\
& \Leftrightarrow n \varphi-1<\frac{p-1}{2}-n \leq n \varphi, n>0 \Leftrightarrow \frac{p-1}{2(1+\varphi)} \leq n<\frac{p+1}{2(1+\varphi)} \\
& \Leftrightarrow-\frac{p+1}{2(1+\varphi)}<-n \leq-\frac{p-1}{2(1+\varphi)} .
\end{aligned}
$$

Now the number of $-n$, which is also the number of $n$, is equal to

$$
\left[-\frac{p-1}{2(1+\varphi)}\right]-\left[-\frac{p+1}{2(1+\varphi)}\right]
$$

Hence,

$$
T(p)=\left[-\frac{p-1}{2(1+\varphi)}\right]-\left[-\frac{p+1}{2(1+\varphi)}\right]
$$

Since

$$
0 \leq\left(-\frac{p-1}{2(1+\varphi)}\right)-\left(-\frac{p+1}{2(1+\varphi)}\right)=\frac{1}{1+\varphi}<1
$$

we have

$$
T(p)=0 \text { or } 1 .
$$

Now we study the sum

$$
\sum_{x<p \leq 2 x} T(p) \log p
$$

We have

$$
\begin{aligned}
\sum_{x<p \leq 2 x} T(p) \log p & =\sum_{x<p \leq 2 x}\left(\left[-\frac{p-1}{2(1+\varphi)}\right]-\left[-\frac{p+1}{2(1+\varphi)}\right]\right) \log p \\
& \leq \sum_{x<m \leq 2 x}\left(\left[-\frac{m-1}{2(1+\varphi)}\right]-\left[-\frac{m+1}{2(1+\varphi)}\right]\right) \Lambda(m)
\end{aligned}
$$




$$
\begin{aligned}
= & \sum_{x<m \leq 2 x}\left(\left(-\frac{m-1}{2(1+\varphi)}-\frac{1}{2}\right)-\left(\left(-\frac{m-1}{2(1+\varphi)}\right)\right)\right) \Lambda(m) \\
& -\sum_{x<m \leq 2 x}\left(\left(-\frac{m+1}{2(1+\varphi)}-\frac{1}{2}\right)-\left(\left(-\frac{m+1}{2(1+\varphi)}\right)\right)\right) \Lambda(m) \\
= & \frac{1}{1+\varphi} \sum_{x<m \leq 2 x} \Lambda(m)+\sum_{x<m \leq 2 x}((\alpha m+\alpha)) \Lambda(m) \\
& -\sum_{x<m \leq 2 x}((\alpha m-\alpha)) \Lambda(m) .
\end{aligned}
$$

The prime number theorem yields

$$
\frac{1}{1+\varphi} \sum_{x<m \leq 2 x} \Lambda(m) \sim \frac{x}{1+\varphi} .
$$

By the formula on page 254 of [2],

$$
((t))=-\sum_{1 \leq|h| \leq x} \frac{e(h t)}{2 \pi i h}+O\left(\min \left(1, \frac{1}{x\|t\|}\right)\right) .
$$

Hence,

$$
\text { (8) } \begin{aligned}
\sum_{x<m \leq 2 x}((\alpha m+\alpha)) \Lambda(m)= & -\sum_{x<m \leq 2 x}\left(\sum_{1 \leq|h| \leq x} \frac{e(\alpha h m)}{2 \pi i h} e(\alpha h)\right) \Lambda(m) \\
& +O\left(\sum_{x<m \leq 2 x} \min \left(1, \frac{1}{x\|\alpha m+\alpha\|}\right) \Lambda(m)\right) \\
\ll & \sum_{1 \leq h \leq x} \frac{1}{h}\left|\sum_{x<m \leq 2 x} \Lambda(m) e(\alpha h m)\right| \\
& +O\left(\log x \sum_{x<m \leq 2 x} \min \left(1, \frac{1}{x\|\alpha m+\alpha\|}\right)\right) .
\end{aligned}
$$

When $J \leq 2 x$, Theorem 1 in [3] states

$$
\sum_{1 \leq h \leq J}\left|\sum_{x<m \leq 2 x} \Lambda(m) e(\alpha h m)\right| \ll x^{\delta}\left(J x / \sqrt{q}+J x^{3 / 4}+(J q x)^{1 / 2}+J^{3 / 5} x^{4 / 5}\right) .
$$

It follows that

$$
\begin{aligned}
\sum_{1 \leq h \leq x} \frac{1}{h} \mid \sum_{x<m \leq 2 x} \Lambda(m) & e(\alpha h m) \mid \\
& \ll \log x \max _{J \leq x} \sum_{J \leq h \leq 2 J} \frac{1}{h}\left|\sum_{x<m \leq 2 x} \Lambda(m) e(\alpha h m)\right| \\
& \ll x^{1-2 \delta} .
\end{aligned}
$$


By Lemma 1 in [3],

$$
\begin{aligned}
\sum_{x<m \leq 2 x} \min \left(1, \frac{1}{x\|\alpha m+\alpha\|}\right) & =\frac{1}{x} \sum_{x<m \leq 2 x} \min \left(x, \frac{1}{\|\alpha m+\alpha\|}\right) \\
& \ll \frac{1}{x}\left(\frac{x^{2}}{q}+x+(x+q) \log q\right) \\
& \ll x^{1-2 \delta} .
\end{aligned}
$$

The combination of (8), (9) and (10) produces

$$
\sum_{x<m \leq 2 x}((\alpha m+\alpha)) \Lambda(m)=O\left(x^{1-\delta}\right) .
$$

In the same way,

$$
\sum_{x<m \leq 2 x}((\alpha m-\alpha)) \Lambda(m)=O\left(x^{1-\delta}\right) .
$$

It follows from (6), (7), (11) and (12) that

$$
\sum_{x<p \leq 2 x} T(p) \log p \leq \frac{1+\delta}{1+\varphi} \cdot x .
$$

Hence,

$$
\sum_{x<p \leq 2 x} T(p) \leq \frac{1+\delta}{1+\varphi} \cdot \frac{x}{\log x}
$$

Thus we have

$$
\sum_{\substack{x<p \leq 2 x \\ T(p)=0}} 1=\sum_{x<p \leq 2 x} 1-\sum_{x<p \leq 2 x} T(p) \geq \frac{\varphi-\varepsilon}{1+\varphi} \cdot \frac{x}{\log x},
$$

so the Theorem is proved.

Acknowledgements. I would like to express my sincere thanks to Professor Yiming Long for his very kind suggestion and helpful discussion. I also thank Academia Sinica and the Natural Science Foundation of China for their support.

\section{References}

[1] Y. Long, Precise iteration formulae of the Maslov-type index theory and ellipticity of closed characteristics, Adv. Math. 154 (2000), 76-131.

[2] H. L. Montgomery and R. C. Vaughan, The distribution of squarefree numbers, in: Recent Progress in Analytic Number Theory, Vol. 1, H. Halberstam and C. Hooley (eds.), Academic Press, London, 1981, 247-256.

[3] R. C. Vaughan, On the distribution of ap modulo 1, Mathematika 24 (1977), 135-141. 
[4] R. C. Vaughan, The Hardy-Littlewood Method, 2nd ed., Cambridge Univ. Press, 1997.

Institute of Mathematics

Academia Sinica

Beijing 100080, P.R. China

E-mail: jiach@math.ac.cn

Received on 14.4.2005

and in revised form on 12.1.2006 